\title{
STRUCTURE OF SPACES OF GERMS OF HOLOMORPHIC FUNCTIONS
}

\author{
N. Van Khue and P. Thien Danh
}

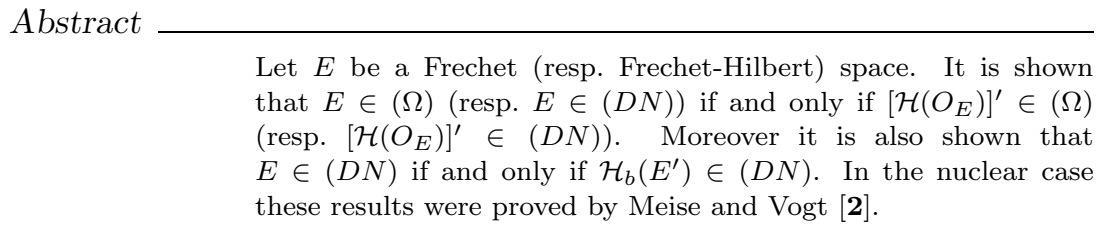

\section{Preliminaries}

1.1. Let $K$ be a compact set in a Frechet space $E$. By $\mathcal{H}(K)$ we denote the space of germs of holomorphic functions on $K$. This space is equipped with the inductive topology

$$
\mathcal{H}(K)=\operatorname{limind}_{U \downarrow K} \mathcal{H}^{\infty}(U)
$$

Here for each neighborhood $U$ of $K$, by $\mathcal{H}^{\infty}(U)$ we denote the Banach space of bounded holomorphic functions on $U$ with the sup-norm

$$
\|f\|_{U}=\sup \{\mid f(z): z \in U\} .
$$

1.2. Let $E^{\prime}$ denote the strong dual space of a Frechet space $E$. A holomorphic function on $E^{\prime}$ is said to be of bounded type if it is bounded on every bounded set in $E^{\prime}$. By $\mathcal{H}_{b}\left(E^{\prime}\right)$ we denote the metric locally convex space of entire functions of bounded type on $E^{\prime}$ equipped with the topology the convergence on bounded sets in $E^{\prime}$.

For more details concerning holomorphic functions on locally convex spaces we refer to the book of Dineen [1]. 
1.3. Assume the topology of $E$ is defined by an increasing fundamental system of seminorms $\left\{\|\cdot\|_{k}\right\}_{k=1}^{\infty}$. For each subset $B$ of $E$, define the generalized seminorm $\|\cdot\|_{B}^{*}: E^{\prime} \rightarrow[0,+\infty]$, by

$$
\|u\|_{B}^{*}=\sup \{|u(x)|: x \in B\}
$$

Write $\|\cdot\|_{q}^{*}$ for $B=U_{q}=\left\{x \in E:\|x\|_{q} \leq 1\right\}$.

Using this notation define $E$ to have the property

$$
\begin{aligned}
& (D N): \quad \exists p \forall q \exists k, \quad C>0:\|\cdot\|_{q}^{2} \leq C\|\cdot\|_{k}\|\cdot\|_{p} . \\
& (\Omega): \forall p \exists q \forall k \exists d, \quad C>0:\|\cdot\|_{q}^{* 1+d} \leq C\|\cdot\|_{k}^{*}\|\cdot\|_{p}^{* d} .
\end{aligned}
$$

The properties $(D N),(\Omega)$ and the other many properties were introduced and investigated by Vogt (see, for example, $[\mathbf{7}],[8]$, etc.). In $[\mathbf{8}]$ he has proved that $E \in(D N)$ (resp. $E \in(\Omega)$ ) if and only if $E$ is isomorphic to a subspace (a quotient space) of the space $B \hat{\otimes}_{\pi} s$ for some Banach space $B$, where $s$ is the space of rapidly decreasing sequences.

The following three theorems are proved in the present paper.

Theorem 1. Let $E$ be a Frechet space. Then the following are equivalent

(i) $E \in(\Omega)$

(ii) $[\mathcal{H}(K)]^{\prime} \in(\Omega)$ for some non-empty compact set $K$ in $E$.

(iii) $[\mathcal{H}(K)]^{\prime} \in(\Omega)$ for all compact sets $K$ in $E$.

Theorem 2. Let $E$ be a Frechet-Hilbert space. Then $E \in(D N)$ if and only if $\left[\mathcal{H}\left(O_{E}\right)\right]^{\prime} \in(D N)$.

Theorem 3. Let $E$ be a Frechet space. Then $E \in(D N)$ if and only if $\mathcal{H}_{b}\left(E^{\prime}\right) \in(D N)$.

The proofs of Theorems 1, 2 and 3 are presented in Sections 2, 3 and 4 respectively. 


\section{Proof of Theorem 1}

2.1. Lemma. Let $E$ be a Frechet space. Then $E \in(\Omega)$ if and only if $E^{\prime}$ is isomorphic to a subspace of $B \hat{\otimes}_{\pi} s^{\prime}$ for some Banach space $B$.

Proof: Suppose $E^{\prime}$ is isomorphic to a subspace of the space $B \hat{\otimes}_{\pi} s^{\prime}$ where $B$ is some Banach space. Then $E^{\prime \prime}$ is isomorphic to a quotient space of $\left(B \hat{\otimes}_{\pi} s^{\prime}\right)^{\prime} \cong B^{\prime} \hat{\otimes}_{\pi} s$ and hence $E^{\prime \prime} \in(\Omega)$. This implies that $E \in(\Omega)$ since

$$
\|u\|_{k}^{*}=\sup \left\{|v(u)|: v \in U_{k}^{00} \subset E^{\prime \prime}\right\} \text { for } u \in E^{\prime} .
$$

Conversely assume that $E \in(\Omega)$. Consider the canonical resolution

$$
0 \longrightarrow E \longrightarrow \prod_{k \geq 1} E_{k} \stackrel{R}{\longrightarrow} \prod_{k \geq 1} E_{k} \longrightarrow 0
$$

as constructed by Palamodov [4], where for each $k \geq 1, E_{k}$ stands for the Banach space associated to $\|\cdot\|_{k}$.

Since $E$ is isomorphic to a quotient space of $B \hat{\otimes}_{\pi} s$ with $B$ is some Banach space $[\boldsymbol{8}], E$ is quasinormable. Hence we may assume that every bounded set in $E_{k+1}$ can be approximated by a bounded set in $E_{k+2}$ under the canonical map $E_{k+2} \rightarrow E_{k}$. It follows from [4] that every bounded set in $\prod_{k>1} E_{k}$ is the image of a bounded set in $\prod_{k>1} E_{k}$ under $R$. By modifying the argument in $[8]$ we imply that $E$ is isomorphic to a quotient space of $B \hat{\otimes}_{\pi} s$ for which $E^{\prime}$ is isomorphic to a suspace of $\left(B \hat{\otimes}_{\pi} s\right)^{\prime} \cong B^{\prime} \hat{\otimes}_{\pi} s^{\prime}$.

The following lemma is an immediate consequence of the preceding lemma.

2.2. Lemma. Let $E$ be a Frechet space. Then $E \in(\Omega)$ if and only if $E^{\prime \prime} \in(\Omega)$.

2.3. Lemma. Let $B$ be a Banach space. Then $\left[\mathcal{H}\left(O_{B \hat{\otimes}_{\pi} s}\right)\right]^{\prime} \in(\Omega)$.

Proof: Let $\left\{e_{j}\right\}$ be the canonical basis of $s$ with the dual basis $\left\{e_{j}^{*}\right\}$ of $s^{\prime}$. Since $s$ is nuclear without loss of generality we may assume that 


$$
\delta_{p}=\sum_{j \geq 1}\left\|e_{j}^{*}\right\|_{p+1}^{*}\left\|e_{j}\right\|_{p}<1 / e \text { for } p \geq 1
$$

For each $p \geq 1$, put

$$
\begin{array}{r}
\|f\|_{p+1}=\sup \left\{\left(\frac{1}{p+1}\right)^{n} \sum_{j_{1}, \ldots, j_{n} \geq 1}\left|\widehat{P_{n} f}\left(u_{1} \otimes e_{j_{1}}, \ldots, u_{n} \otimes e_{j_{n}}\right)\right|\right. \\
\left.\quad \times\left\|e_{j_{1}}^{*}\right\|_{p+1}^{*} \ldots\left\|e_{j_{n}}^{*}\right\|_{p+1}^{*}: u_{1}, \ldots, u_{n} \in W, n \geq 0\right\}
\end{array}
$$

for $f \in \mathcal{H}^{\infty}\left(\operatorname{conv}\left(W \otimes U_{p}\right)\right)$, where $W$ is the unit ball of $B$ and

$$
f(\omega)=\sum_{n \geq 0} P_{n} f(\omega), \quad \omega \in \operatorname{conv}\left(W \otimes U_{p}\right)
$$

is the Taylor expansion of $f$ at $0 \in B \hat{\otimes}_{\pi} s$.

Since

$$
\begin{aligned}
\|\mid f\|_{p+1} & =\sup \left\{\left(\frac{1}{p+1}\right)^{n} \sum_{j_{1}, \ldots, j_{n} \geq 1}\left|\widehat{P_{n} f}\left(u_{1} \otimes \frac{e_{j_{1}}}{\left\|e_{j_{1}}\right\|_{p}}, \ldots, u_{n} \otimes \frac{e_{j_{n}}}{\left\|e_{j_{n}}\right\|_{p}}\right)\right|\right. \\
& \left.\times\left\|e_{j_{1}}^{*}\right\|_{p+1}^{*} \ldots\left\|e_{j_{n}}\right\|_{p} \ldots\left\|e_{j_{1}}^{*}\right\|_{p+1}^{*}\left\|e_{j_{1}}\right\|_{p}: u_{1}, \ldots, u_{n} \in W, n \geq 0\right\} \\
& \leq C_{p}\|f\|_{\operatorname{conv}\left(W \otimes U_{p}\right)}
\end{aligned}
$$

for $f \in \mathcal{H}^{\infty}\left(\operatorname{conv}\left(W \otimes U_{p}\right)\right)$, where

$$
C_{p}=\sup \left\{\left(\frac{\delta_{p}}{p+1}\right)^{n} \frac{n^{n}}{n !}: n \geq 0\right\}<\infty
$$

it follows that $\left\||\cdot \||_{p+1}\right.$ is continuous on $\mathcal{H}^{\infty}\left(\operatorname{conv}\left(W \otimes U_{p}\right)\right)$. 
StruCture of SPaCeS OF GERMS OF HOLOMORPhiC FUnCTIONS 471

On the other hand, we have

$$
\begin{aligned}
& \|f\|_{\operatorname{conv}\left(W \otimes \frac{U_{p+1}}{p+2}\right)}=\sup \left\{\left|f\left(\frac{1}{p+2} \sum_{k \geq 1} \lambda_{k} u_{k} \otimes v_{k}\right)\right|\right. \\
& \left.: u_{k} \in W, v_{k} \in U_{p+1}, \sum_{k \geq 1}\left|\lambda_{k}\right| \leq 1\right\} \\
& \leq \sup \left\{\sum_{n \geq 0}\left(\frac{p+1}{p+2}\right)^{n} \sum_{k_{1}, \ldots, k_{n} \geq 1}\left|\lambda_{k_{1}}\right| \ldots\left|\lambda_{k_{n}}\right| \sum_{j_{1}, \ldots, j_{n} \geq 1}\left(\frac{1}{p+1}\right)^{n}\right. \\
& \times\left|\widehat{P_{n} f}\left(u_{k_{1}} \otimes e_{j_{1}}, \ldots, u_{k_{n}} \otimes e_{j_{n}}\right)\right|\left|e_{j_{1}}^{*} v_{k_{1}}\right| \ldots\left|e_{j_{n}}^{*}\left(v_{k_{n}}\right)\right| \\
& \left.: u_{k} \in W, v_{k} \in U_{p+1}, \sum_{k \geq 1}\left|\lambda_{k}\right| \leq 1\right\} \\
& \leq \sup \left\{\sum_{n \geq 0}\left(\frac{p+1}{p+2}\right)^{n} \sum_{k_{1}, \ldots, k_{n} \geq 1}\left|\lambda_{k_{1}}\right| \ldots\left|\lambda_{k_{n}}\right| \sum_{j_{1}, \ldots, j_{n} \geq 1}\left(\frac{1}{p+1}\right)^{n}\right. \\
& \times\left|\widehat{P_{n} f}\left(u_{k_{1}} \otimes e_{j_{1}}, \ldots, u_{k_{n}} \otimes e_{j_{n}}\right)\right|\left\|e_{j_{1}}^{*}\right\|_{p+1}^{*} \ldots\left\|e_{j_{n}}^{*}\right\|_{p+1}^{*} \\
& \left.: u_{k} \in W, \sum_{k \geq 1}\left|\lambda_{k}\right| \leq 1\right\} \\
& \leq \sup \left\{\sum_{n \geq 0}\left(\frac{p+1}{p+2}\right)^{n} \sum_{k_{1}, \ldots, k_{n} \geq 1}\left|\lambda_{k_{1}}\right| \ldots\left|\lambda_{k_{n}}\right||f| \|_{p+1}: \sum_{k \geq 1}\left|\lambda_{k}\right| \leq 1\right\} \\
& \leq\left(\sum_{n \geq 0}\left(\frac{p+1}{p+2}\right)^{n}\right)\|\mid f\|_{p+1} .
\end{aligned}
$$

Hence

$$
\mathcal{H}\left(O_{B \hat{\otimes}_{\pi} s}\right) \cong \operatorname{limind}\left[\mathcal{H}^{\infty}\left(\operatorname{conv}\left(W \otimes U_{p}\right)\right):\left\||\cdot \||_{p+1}\right] .\right.
$$

Given $p \geq 1$, choose $q \geq p$ such that

$$
\forall k \exists C, d>0:\left\|e_{j}^{*}\right\|_{q}^{* 1+d} \leq C\left\|e_{j}^{*}\right\|_{k}^{*}\left\|e_{j}^{*}\right\|_{p}^{* d} \forall j \geq 1 .
$$


Since $\|\cdot\|_{q}^{*} \leq\|\cdot\|_{p}^{*}$, the above inequality holds for every $d^{\prime} \geq d$. Hence we may assume that $C k p^{d} \leq q^{1+d}$, then

$$
\begin{aligned}
& \|\mid f\|_{q}^{1+d}=\sup \left\{\left(\frac{1}{q}\right)^{n} \sum_{j_{1}, \ldots, j_{n} \geq 1}\left|\widehat{P_{n} f}\left(u_{1} \otimes e_{j_{1}}, \ldots, u_{n} \otimes e_{j_{n}}\right)\right|\right. \\
& \left.\times\left\|e_{j_{1}}^{*}\right\|_{q}^{*} \ldots\left\|e_{j_{n}}^{*}\right\|_{q}^{*}: u_{1}, \ldots, u_{n} \in W, n \geq 0\right\}^{1+d} \\
& \leq \sup \left\{\left(\frac{1}{C k p^{d}}\right)^{\frac{n}{1+d}} \sum_{j_{1}, \ldots, j_{n} \geq 1}\left|\widehat{P_{n} f}\left(u_{1} \otimes e_{j_{1}}, \ldots, u_{n} \otimes e_{j_{n}}\right)\right|\right. \\
& \times C^{\frac{n}{1+d}}\left\|e_{j_{1}}^{*}\right\|_{k}^{* 1 / 1+d} \ldots\left\|e_{j_{n}}^{*}\right\|_{k}^{* 1 / 1+d} \\
& \left.\times\left\|e_{j_{1}}^{*}\right\|_{p}^{* d / 1+d} \ldots\left\|e_{j_{n}}^{*}\right\|_{p}^{* d / 1+d}: u_{1}, \ldots, u_{n} \in W, n \geq 0\right\}^{1+d} \\
& \leq \sup \left\{(1 / k)^{n} \sum_{j_{1}, \ldots, j_{n} \geq 1}\left|\widehat{P_{n} f}\left(u_{1} \otimes e_{j_{1}}, \ldots, u_{n} \otimes e_{j_{n}}\right)\right|\right. \\
& \left.\times\left\|e_{j_{1}}^{*}\right\|_{k}^{*} \ldots\left\|e_{j_{n}}^{*}\right\|_{k}^{*}: u_{1}, \ldots, u_{n} \in W, n \geq 0\right\} \\
& \times \sup \left\{(1 / p)^{n} \sum_{j_{1}, \ldots, j_{n} \geq 1}\left|\widehat{P_{n} f}\left(u_{1} \otimes e_{j_{1}}, \ldots, u_{n} \otimes e_{j_{n}}\right)\right|\right. \\
& \left.\times\left\|e_{j_{1}}^{*}\right\|_{p}^{*} \ldots\left\|e_{j_{n}}^{*}\right\|_{p}^{*}: u_{1}, \ldots, u_{n} \in W, n \geq 0\right\}^{d} \\
& =\||f|\|_{k}\||f|\|_{p}^{d}
\end{aligned}
$$

for $f \in \mathcal{H}\left(O_{B \hat{\otimes}_{\pi} s}\right)$.

Since $B \hat{\otimes}_{\pi} s$ is quasinormale, according to Mujica [3] there exists a Frechet space $F$ such that $F^{\prime} \cong \mathcal{H}\left(O_{B \hat{\otimes}_{\pi} s}\right)$. Combining this fact together with the inequality

$$
\left.\left\|\left|\cdot\left\|\left.\right|^{1+d} \leq\right\|\right| \cdot\right\|\right|_{k}\left\||\cdot \||_{p}^{d} \text { on } \mathcal{H}\left(O_{B \hat{\otimes}_{\pi} s}\right),\right.
$$


by virtue of Lemma 2.2 we obtain $\left[\mathcal{H}\left(O_{B \hat{\otimes}_{\pi} s}\right)\right]^{\prime} \cong F^{\prime \prime}$ has $(\Omega)$.

Now we are able to prove Theorem 1.

(iii) $\rightarrow$ (ii) is trivial.

(ii) $\rightarrow$ (i). Fix $x_{0} \in K$. Then the form

$$
\varphi \rightarrow \varphi^{\prime}\left(x_{0}\right)
$$

defines a left inverse of the canonical map $E^{\prime} \rightarrow \mathcal{H}(K)$. Hence $E^{\prime \prime} \in(\Omega)$, this implies that $E \in(\Omega)$.

(i) $\rightarrow$ (iii). Assume that $E \in(\Omega)$. By Vogt [5] there exists a continuous linear map $R$ from $B \hat{\otimes}_{\pi} s$ onto $E$ for some Banach space $B$.

Let $\left\{W_{k}\right\}$ be a neighbourhood basis of $0 \in B \hat{\otimes}_{\pi} s$. Then $\left\{V_{k}=R\left(W_{k}\right)\right\}$ forms a neighbourhood basis of $0 \in E$. Lemma 2.3 gives

$$
\forall p \exists q \exists C, d>0 \forall f \in \mathcal{H}^{\infty}\left(W_{p}\right):\|f\|_{W_{q}}^{1+d} \leq C\|f\|_{W_{k}}\|f\|_{W_{p}}^{d} .
$$

Thus

$$
\|g\|_{V_{q}}^{1+d} \leq\|g\|_{V_{k}}\|g\|_{V_{p}}^{d} \forall g \in \mathcal{H}^{\infty}\left(V_{p}\right) .
$$

Next let $K$ be an arbitrary compact set in $E$. From $(*)$ we deduce that

$$
\|g\|_{K+V_{q}}^{1+d} \leq C\|g\|_{K+V_{p}}\|g\|_{K+V_{p}}^{d} \forall f \in \mathcal{H}^{\infty}\left(K+V_{p}\right) .
$$

According to Mujica $[\mathbf{3}]$ there exists a Frechet space $F$ verifying $F^{\prime} \cong$ $\mathcal{H}(K)$ since $E$ is quasinormale, invoke Lemma 2.2 we conclude that $[\mathcal{H}(K)]^{\prime} \cong F^{\prime \prime} \in(\Omega)$.

\section{Proof of Theorem 2}

3.1. Lemma. Let $E$ be a Frechet-Hilbert space with $E \in(D N)$. Then $E$ is isomorphic to a subspace of the space $l^{2}(I) \hat{\otimes}_{\pi}$ for some index set.

Proof: Choose an index set $I$ such that $E$ is isomorphic to a subspace of $\left[l^{2}(I)\right]^{N}$. Consider the exact sequence of nuclear Frechet spaces

$$
0 \rightarrow s \rightarrow s \rightarrow \omega \rightarrow 0
$$

constructed by Vogt $[\mathbf{8}]$. By tensoring this sequence with $l^{2}(I)$ we get the exact sequence of Frechet-Hilbert spaces [8],

$$
0 \rightarrow l^{2}(I) \hat{\otimes}_{\pi} s \rightarrow l^{2}(I) \hat{\otimes}_{\pi} s \stackrel{q}{\rightarrow}\left[l^{2}(I)\right]^{N} \rightarrow 0
$$


Let $\tilde{E}=q^{-1}(E)$. Since

$$
0 \rightarrow l^{2}(I) \hat{\otimes}_{\pi} s \rightarrow \hat{E} \stackrel{q}{\rightarrow} E \rightarrow 0
$$

is a exact sequence of Frechet-Hilbert spaces in which $l^{2}(I) \hat{\otimes}_{\pi} s \in(\Omega)$ and $E \in(D N)$ by $\operatorname{Vogt}[\mathbf{9}] q$ has a right inverse. Hence $E$ is isomorphic to subspace of $\tilde{E}$ and hence of $l^{2}(I) \hat{\otimes}_{\pi} s$.

3.2. Lemma. Let $B$ be a Banach space. Then $\left[\mathcal{H}\left(O_{B \hat{\otimes}_{\pi} s}\right)\right]^{\prime} \in(D N)$.

Proof: Let $W$ denote the unit ball of $B$. Write the Taylor expansion of each $f \in \mathcal{H}\left(O_{B \hat{\otimes}_{\pi} s}\right)$ at $0 \in B \hat{\otimes}_{\pi} s$

$$
f(\omega)=\sum_{n \geq 0} P_{n} f(\omega) .
$$

Formally we have

$$
\begin{aligned}
f\left(\sum_{k \geq 1} \lambda_{k} u_{k} \otimes v_{k}\right) & =\sum_{n \geq 0} \sum_{k_{1}, \ldots, k_{n} \geq 1} \lambda_{k_{1}} \ldots \lambda_{k_{n}} \\
& \times \sum_{j_{1}, \ldots, j_{n} \geq 1} \widehat{P_{n} f}\left(u_{k_{1}} \otimes e_{j_{1}}, \ldots, u_{k_{n}} \otimes e_{j_{n}}\right) e_{j_{1}}^{*}\left(v_{k_{1}}\right) \ldots e_{j_{n}}^{*}\left(v_{k_{n}}\right)
\end{aligned}
$$

for $\omega=\sum_{k \geq 1} \lambda_{k} u_{k} \otimes v_{k} \in B \hat{\otimes}_{\pi} s$.

For each $p \geq 1$ as in Theorem 1 put

$$
\begin{array}{r}
\|\mid f\|_{p}=\sup \left\{\frac{1}{p^{n}} \sum_{j_{1}, \ldots, j_{n} \geq 1}\left|\widehat{P_{n} f}\left(u_{1} \otimes e_{j_{1}}, \ldots, u_{n} \otimes e_{j_{n}}\right)\right|\left(j_{1} \ldots j_{n}\right)^{-p}\right. \\
\left.: n \geq 0, u_{1}, \ldots, u_{n} \in W\right\}
\end{array}
$$

and

$$
F_{p}=\left\{f \in \mathcal{H}\left(O_{B \hat{\otimes}_{\pi} s}\right):\|\mid f\| \|_{p}<+\infty\right\} .
$$

Then

$$
\mathcal{H}\left(O_{B \hat{\otimes}_{\pi} s}\right) \cong \operatorname{limind}_{p} F_{p}
$$


Structure of SPACES OF GERMS OF HOLOMORPhic FUnCtions 475

In order to prove that $\left[\mathcal{H}\left(O_{B \hat{\otimes}_{\pi} s}\right)\right]^{\prime} \in(D N)$ we check that

$$
\forall q \exists k, C>0: W_{q} \subset C s W_{1}+\frac{1}{s} W_{k} \quad \forall s>0
$$

where for each $q \geq 1$ put

$$
W_{q}=\left\{f \in F_{q}:\|\mid f\|_{q}<1\right\} .
$$

Obviously (4) holds for $0<s \leq 1$. Let $s>1$. Choose $k=q^{3}$.

We have

(3)

$$
\begin{aligned}
& \sup \left\{\frac{1}{k^{n}} \sum_{j_{1}, \ldots, j_{n} \geq 1}\left|\widehat{P_{n} f}\left(u_{1} \otimes e_{j_{1}}, \ldots, u_{n} \otimes e_{j_{n}}\right)\right|\left(j_{1} \ldots j_{n}\right)^{-k}\right. \\
& \left.: n \geq \frac{\log s}{\log \frac{k}{q}}, u_{1}, \ldots, u_{n} \in W\right\} \\
& +\sup \left\{\frac{1}{k^{n}} \sum_{\left(j_{1} \ldots j_{n} \geq s^{\frac{1}{k-q}}\right.}\left|\widehat{P_{n} f}\left(u_{1} \otimes e_{j_{1}}, \ldots, u_{n} \otimes e_{j_{n}}\right)\right|\left(j_{1} \ldots j_{n}\right)^{-k}\right. \\
& \left.\quad: n \geq 0, u_{1}, \ldots, u_{n} \in W\right\} \\
& \leq \sup \left\{\frac{1}{q^{n}} \sum_{j_{1}, \ldots, j_{n} \geq 1}\left|\widehat{P n_{f}}\left(u_{1} \otimes e_{j_{1}}, \ldots, u_{n} \otimes e_{j_{n}}\right)\right|\left(j_{1} \ldots j_{n}\right)^{-q}\right. \\
& \left.\leq \ln \geq 0, u_{1}, \ldots, u_{n} \in W\right\} \\
& \leq \frac{2}{s} \text { for } f \in W_{q}\left\{\left(\frac{q}{k}\right)^{n}: n \geq \frac{\log s}{\log \frac{k}{q}}\right\}+\sup \left\{\left(j_{1} \ldots j_{n}\right)^{q-k}:\left(j_{1} \ldots j_{n}\right) \geq s^{\frac{1}{k-q}}\right\}
\end{aligned}
$$


and

$$
\begin{aligned}
& \sup \left\{\sum_{j_{1} \ldots j_{n} \leq s^{\frac{1}{k-q}}}\left|\widehat{P_{n} f}\left(u_{1} \otimes e_{j_{1}}, \ldots, u_{n} \otimes e_{j_{n}}\right)\right|\left(j_{1} \ldots j_{n}\right)^{-1}\right. \\
& \left.: n \leq \frac{\log s}{\log \frac{k}{q}}, u_{1}, \ldots, u_{n} \in W\right\} \\
& \leq\left\||f \||_{q} \sup \left\{q^{n}\left(j_{1} \ldots j_{n}\right)^{q}: n \leq \frac{\log s}{\log \frac{k}{q}}, j_{1} \ldots j_{n} \leq s^{\frac{1}{k-q}}\right\}\right. \\
& \leq q^{\frac{\log s}{\log \frac{k}{q}} s^{\frac{1}{q}} \leq s \text { for } f \in W_{q} .}
\end{aligned}
$$

From (3) and (4) it follows that

$$
W_{q} \subset s W_{1}+\frac{2}{s} W_{k} \quad \forall s>0 .
$$

Now by Lemma 2.1 and 2.2 we can complete the proof of Theorem 2 . Indeed, let $E$ be a Frechet-Hilbert space with $(D N)$. By Lemma 2.1, $E$ can be considered as a subspace of the space $l^{2}(I) \hat{\otimes}_{\pi} s$ for some index $I$. Since $l^{2}(I) \hat{\otimes}_{\pi} s$ has a fundamental system of Hilbert semi-norms, the restriction map $R: \mathcal{H}\left(O_{l^{2}(I) \hat{\otimes}_{\pi} s}\right) \rightarrow \mathcal{H}\left(O_{E}\right)$ is surjective. Moreover it is easy to check that every bounded set in $\mathcal{H}\left(O_{E}\right)$ is the image of a bounded set in $\mathcal{H}\left(O_{l^{2}(I) \hat{\otimes}_{\pi} s}\right)$ because of the regularity of $\mathcal{H}\left(O_{E}\right)$ (see [1] $)$. Thus $R^{\prime}:\left[\mathcal{H}\left(O_{E}\right)\right]^{\prime} \rightarrow\left[\mathcal{H}\left(O_{B \hat{\otimes}_{\pi} s}\right)\right]^{\prime}$ is isomorphic onto image. Hence, by Lemma 2.2 it follows that $\left[\mathcal{H}\left(O_{E}\right)\right]^{\prime} \in(D N)$. Conversely, assume that $\left[\mathcal{H}\left(O_{E}\right) \in(D N)\right.$. Since the form

$$
\mathcal{H}\left(O_{E}\right) \ni f \mapsto f^{\prime}(0) \in E^{\prime}
$$

defines a continuous linear map from $\mathcal{H}\left(O_{E}\right)$ onto $E^{\prime}$ which is a left inverse of the canonical map $E^{\prime} \rightarrow \mathcal{H}\left(O_{E}\right)$, it follows that $E^{\prime \prime}$ is isomorphic to a subspace of $\left[\mathcal{H}\left(O_{E}\right)\right]^{\prime}$. Hence $E^{\prime \prime} \in(D N)$. On the other hand, since $E$ is reflexive, $E \cong E^{\prime \prime} \in(D N)$.

\section{Proof of Theorem 3}

By Vogt $[8] E$ can be considered as a subspace of $B \hat{\otimes}_{\pi} s$, where $B$ is some Banach space. Since every bounded set in $E^{\prime}$ can be lifted from $E^{\prime}$ 
to $\left(B \hat{\otimes}_{\pi} s\right)^{\prime} \cong B^{\prime} \hat{\otimes}_{\pi} s^{\prime}$ under the restriction map $R:\left(B \hat{\otimes}_{\pi} s\right)^{\prime} \rightarrow E^{\prime}$, it follows that $\mathcal{H}_{b}\left(E^{\prime}\right)$ is isomorphic to a subspace of $\mathcal{H}_{b}\left(\left(B \hat{\otimes}_{\pi} s\right)\right)^{\prime}$. Thus it remains to check that $\mathcal{H}\left(\left(B \hat{\otimes}_{\pi} s\right)^{\prime}\right) \cong \mathcal{H}_{b}\left(B^{\prime} \hat{\otimes}_{\pi} s^{\prime}\right) \in(D N)$.

Given $p \geq 1$. Take $q>p$ such that

$$
\delta=\sum_{j \geq 1}\left\|e_{j}^{*}\right\|_{q}^{*}\left\|e_{j}\right\|_{p}<\frac{1}{e^{2} p},
$$

where

$$
\|u\|_{q}^{*}=\sup \left\{|u(x)|:\|x\|_{q} \leq 1\right\}, \quad u \in s^{\prime} .
$$

Let $W$ denote the unit ball of $B^{\prime}$. Then for every $f \in \mathcal{H}\left(B^{\prime} \hat{\otimes}_{\pi} s^{\prime}\right)$ we have

$$
\begin{aligned}
& \sup \left\{\sum_{j_{1}, \ldots, j_{n} \geq 1} p^{n}\left|\widehat{P_{n} f}\left(u_{1} \otimes e_{j_{1}}^{*}, \ldots, u_{n} \otimes e_{j_{n}}^{*}\right)\right|\left\|e_{j_{1}}\right\|_{p} \ldots\left\|e_{j_{n}}\right\|_{p}\right. \\
& \left.: u_{1}, \ldots, u_{n} \in W, n \geq 0\right\} \\
& =\sup \left\{\sum_{j_{1}, \ldots, j_{n} \geq 1} p^{n}\left|\widehat{P_{n} f}\left(u_{1} \otimes \frac{e_{j_{1}}^{*}}{\left\|e_{j_{1}}^{*}\right\|_{q}^{*}}, \ldots, u_{n} \otimes \frac{e_{j_{n}}^{*}}{\left\|e_{j_{n}}^{*}\right\|_{q}^{*}}\right)\right|\right. \\
& \left.\times\left\|e_{j_{1}}^{*}\right\|_{q}^{*}\left\|e_{j_{1}}\right\|_{p} \ldots\left\|e_{j_{n}}^{*}\right\|_{q}^{*}\left\|e_{j_{n}}\right\|_{p}: u_{1}, \ldots, u_{n} \in W, n \geq 0\right\} \\
& \leq \sup \left\{\frac{n^{n} p^{n}}{n !} \sum_{j_{1}, \ldots, j_{n} \geq 1}\left\|e_{j_{1}}^{*}\right\|_{q}^{*}\left\|e_{j_{1}}\right\|_{p} \ldots\left\|e_{j_{n}}^{*}\right\|_{q}^{*}\left\|e_{j_{n}}\right\|_{p}: n \geq 0\right\} \\
& \times\|f\|_{\operatorname{conv}\left(W \otimes U_{q}^{0}\right)} \\
& \leq \sup \frac{n^{n} p^{n}}{n !}\left(\frac{1}{e^{2} p}\right)^{n}\|f\|_{\operatorname{conv}\left(W \otimes U_{q}^{0}\right)} \\
& =C(p)\|f\|_{\operatorname{conv}\left(W \otimes U_{q}^{0}\right)}
\end{aligned}
$$

where

$$
C(p)=\sup \frac{n^{n} p^{n}}{n !}\left(\frac{1}{e^{2} p}\right)^{n}<\infty
$$


and $f(\omega)=\sum_{n \geq 0} P_{n} f(\omega)$ is the Taylor expansion of $f$ at $0 \in B^{\prime} \hat{\otimes}_{\pi} s^{\prime}$.

Thus the form (1) defines a continuous semi-norms $\left\||\cdot \||_{p}\right.$ on $\mathcal{H}_{b}\left(B^{\prime} \hat{\otimes}_{\pi} s^{\prime}\right)$. On the other hand, since

$$
\begin{aligned}
& \|f\|_{\operatorname{conv}\left(W \otimes U_{p}^{0}\right)}=\sup \left\{\left|f\left(\sum_{k \geq 1} \lambda_{k} u_{k} \otimes v_{k}\right)\right|: u_{k} \in W, v_{k} \in U_{p}^{0}, \sum_{k \geq 1}\left|\lambda_{k}\right| \leq 1\right\} \\
& \leq \sup \left\{\sum_{n \geq 0} \frac{1}{p^{n}} \sum_{k_{1}, \ldots, k_{n} \geq 1}\left|\lambda_{k_{1}}\right| \ldots\left|\lambda_{k_{n}}\right|\right. \\
& \times \sum_{j_{1}, \ldots, j_{n} \geq 1} p^{n}\left|\widehat{P_{n} f}\left(u_{k_{1}} \otimes e_{j_{1}}^{*}, \ldots, u_{k_{n}} \otimes e_{j_{n}}^{*}\right)\right| \\
& \left.\times\left\|e_{j_{1}}^{*}\left(v_{k_{1}}\right)\right\| \ldots\left\|e_{j_{n}}^{*}\left(v_{k_{n}}\right)\right\|: u_{k} \in W, v_{k} \in U_{p}^{0}, \sum_{k \geq 1}\left|\lambda_{k}\right| \leq 1\right\} \\
& \leq \sup \left\{\sum_{n \geq 0} \frac{1}{p^{n}} \sum_{k_{1}, \ldots, k_{n} \geq 1}\left|\lambda_{k_{1}}\right| \ldots\left|\lambda_{k_{n}}\right|\right. \\
& \times \sum_{j_{1}, \ldots, j_{n} \geq 1} p^{n}\left|\widehat{P_{n} f}\left(u_{k_{1}} \otimes e_{j_{1}}^{*}, \ldots, u_{k_{n}} \otimes e_{j_{n}}^{*}\right)\right| \\
& \left.\times\left\|e_{j_{1}}^{*}\right\|_{p} \ldots\left\|e_{j_{n}}^{*}\right\|_{p}: u_{k} \in W, v_{k} \in U_{p}^{0}, \sum_{k \geq 1}\left|\lambda_{k}\right| \leq 1\right\} \\
& \leq\left.\sup \left\{\sum_{n \geq 0} \frac{1}{p^{n}} \sum_{k_{1}, \ldots, k_{n} \geq 1}\left|\lambda_{k_{1}}\right| \ldots\left|\lambda_{k_{n}}\right|: \sum_{k \geq 1}\left|\lambda_{k}\right| \leq 1\right\}\|f\|\right|_{p} \\
& \leq\left(\sum_{n \geq 0} \frac{1}{p^{n}}\right)\|\mid f\|_{p} \text { for } f \in \mathcal{H}_{b}\left(B^{\prime} \hat{\otimes}_{\pi} s^{\prime}\right) .
\end{aligned}
$$

it follows that the topology of $\mathcal{H}\left(B^{\prime} \hat{\otimes}_{\pi} s^{\prime}\right)$ can be defined by the system of the semi-norms $\left\{\|\left.|\cdot|\right|_{p}\right\}$. Choose $p \geq 1$ such that $(D N)$ is satisfied. Then

$$
\forall q \exists k \forall j \geq 1:\left\|e_{j}\right\|_{q}^{2} \leq\left\|e_{j}\right\|_{k}\left\|e_{j}\right\|_{p} .
$$

Given $q$. Choose $k$ such that the above condition holds and

$$
q^{2} \leq k p
$$


Structure of SPACES OF GERMS OF HOLOMORPhic FUnCtions 479

Then

$$
\begin{aligned}
& \left\||f \||_{q}^{2}=\sup \left\{q^{n} \sum_{j_{1}, \ldots, j_{n} \geq 1}\left|\widehat{P_{n} f}\left(u_{1} \otimes e_{j_{1}}^{*}, \ldots, u_{n} \otimes e_{j_{n}}^{*}\right)\right|\left\|e_{j_{1}}\right\|_{q} \ldots\left\|e_{j_{n}}\right\|_{q}\right.\right. \\
& \left.: u_{1}, \ldots, u_{n} \in W, n \geq 0\right\}^{2} \\
& \leq \sup \left\{k^{\frac{n}{2}} p^{\frac{n}{2}} \sum_{j_{1}, \ldots, j_{n} \geq 1}\left|\widehat{P_{n} f}\left(u_{1} \otimes e_{j_{1}}^{*}, \ldots, u_{n} \otimes e_{j_{n}}^{*}\right)\right|\right. \\
& \left.\times\left\|e_{j_{1}}\right\|_{k}^{\frac{1}{2}} \ldots\left\|e_{j_{n}}\right\|_{k}^{\frac{1}{2}}\left\|e_{j_{1}}\right\|_{p}^{\frac{1}{2}} \ldots\left\|e_{j_{n}}\right\|_{p}^{\frac{1}{2}}: u_{1}, \ldots, u_{n} \in W, n \geq 0\right\}^{2} \\
& \leq \sup \left\{k^{n} \sum_{j_{1}, \ldots, j_{n} \geq 1}\left|\widehat{P_{n} f}\left(u_{1} \otimes e_{j_{1}}^{*}, \ldots, u_{n} \otimes e_{j_{n}}^{*}\right)\right|\left\|e_{j_{1}}\right\|_{k} \ldots\left\|e_{j_{n}}\right\|_{k}\right. \\
& \left.: u_{1}, \ldots, u_{n} \in W, n \geq 0\right\} \\
& \times \sup \left\{p^{n} \sum_{j_{1}, \ldots, j_{n} \geq 1}\left|\widehat{P_{n} f}\left(u_{1} \otimes e_{j_{1}}^{*}, \ldots, u_{n} \otimes e_{j_{n}}^{*}\right)\right|\left\|e_{j_{1}}\right\|_{p} \ldots\left\|e_{j_{n}}\right\|_{p}\right. \\
& \left.: u_{1}, \ldots, u_{n} \in W, n \geq 0\right\} \\
& =\left\|\left|f\left\|\left.\right|_{k}\right\|\right| f\right\|_{p} \text { for } f \in \mathcal{H}_{b}\left(B^{\prime} \hat{\otimes}_{\pi} s^{\prime}\right) .
\end{aligned}
$$

Consequently $\mathcal{H}_{b}\left(B^{\prime} \hat{\otimes}_{\pi} s^{\prime}\right) \in(D N)$.

Acknowledgements. We would like to express our thanks to the referee for his helpful remarks and suggestions.

\section{References}

1. S. DineEn, "Complex analysis in locally convex spaces," NorthHolland Math. Studies 57, 1981. 
2. R. Meise and D. Vogt, Structure of spaces of holomorphic functions on infinite dimensional polydiscs, Studia Math. 75 (1983), 235-253.

3. J. MujicA, A completeness criterion for inductive limits of Banach spaces, in "Functional Analysis Holomorphy and Approximation Theory II," (G. I. Zapata, ed.), North-Holland Math. Studies 68, 1984, pp. 319-329.

4. V. P. Palamodov, The projective limit functor in the category of linear topological spaces, Mat. Sb. 75 (1968), 567-603 (in Russian).

5. A. Pietsch, "Nuclear Locally Convex Spaces," Ergeb MathGrenzgeb 66, Springer-Verlag, 1972.

6. H. Schaefer, "Topological Vector Spaces," Springer-Verlag, 1971.

7. D. Vogt, Frechetraume zwischen dene jede stetige linear Abbildung beschrankt ist, J. Reine Angew. Math. 345 (1983), 182-200.

8. D. Vogt, On two classes of F-spaces, Arch. Math. 45 (1985), 255-266.

9. D. Vogt, Interpolation of nuclear operators and a spliting theorem for exact sequences of Frechet spaces, Preprint.

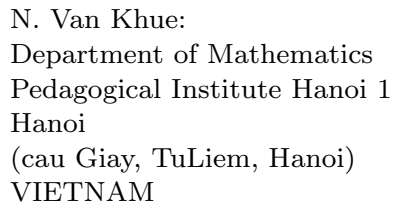

P. Thien Danh:

Department of Mathematics

College of Education

280 An Duong Vuong, District 5

Ho Chi Minh City

VIETNAM

Primera versió rebuda el 17 d'Abril de 1996, darrera versió rebuda el 21 d'Abril de 1997 